\title{
Temperature dependent carrier lifetime studies of Mo in crystalline silicon
}

\author{
Bijaya B. Paudyal, ${ }^{1, a)}$ Keith R. McIntosh, ${ }^{1}$ Daniel H. Macdonald, ${ }^{2}$ and Gianluca Coletti ${ }^{3}$ \\ ${ }^{1}$ Centre for Sustainable Energy Systems, Australian National University, Canberra, Australian \\ Capital Territory 0200, Australia \\ ${ }^{2}$ Department of Engineering, College of Engineering and Computer Science, Australian National University, \\ Canberra, Australian Capital Territory 0200, Australia \\ ${ }^{3}$ ECN Solar Energy, Energy research Centre of Netherlands, NL-1755 LE Petten, The Netherlands
}

(Received 18 November 2009; accepted 12 January 2010; published online 9 March 2010)

\begin{abstract}
The capture cross sections of both electrons $\sigma_{n}$ and holes $\sigma_{p}$ were determined for interstitial molybdenum in crystalline silicon over the temperature range of -110 to $150{ }^{\circ} \mathrm{C}$. Carrier lifetime measurements were performed on molybdenum-contaminated silicon using a temperature controlled photoconductance instrument. Injection dependent lifetime spectroscopy was applied at each temperature to calculate $\sigma_{p}$ and $\sigma_{n}$. This analysis involved a novel approach that independently determined the capture cross sections at each temperature assuming a known defect density and thermal velocity. Since the energy state is in the lower half of the bandgap, the determination of $\sigma_{p}$ is unaffected by the defect energy at all temperatures, and $\sigma_{p}$ is found to decrease with temperature in a fashion consistent with excitonic Auger capture. At temperatures below $0{ }^{\circ} \mathrm{C}$, the determination of $\sigma_{n}$ is also unaffected by the defect energy due to the suppression of thermal emission, and $\sigma_{n}$ decreases with temperature as well. It is shown that a projection of $\sigma_{n}$ to higher temperature suggests the defect has an energy of $0.375 \mathrm{eV}$ above the valance band edge of silicon. (C) 2010 American Institute of Physics. [doi:10.1063/1.3309833]
\end{abstract}

\section{INTRODUCTION}

Molybdenum (Mo) is a transition metal and a potential source of contamination in silicon devices. ${ }^{1}$ Mo remains in interstitial form within the lattice structure of silicon and creates an electrically active defect. Istratov et $a l^{2}$ measured significant amounts of Mo in commercially available multicrystalline silicon for solar cells, while Davis et al. ${ }^{3}$ showed Mo to have a very strong impact on solar cell efficiency in $\mathrm{Cz}$ silicon, reducing it by up to one third.

In 1995, Graff ${ }^{1}$ summarized published information about the electrical properties of Mo in silicon, mostly based on deep level transient spectroscopy (DLTS). Interstitial Mo was found to introduce a single defect energy level $\left(E_{t}\right)$ that is donorlike and exists at $0.28 \pm 0.01 \mathrm{eV}$ above the valance band edge of silicon $\left(E_{V}\right)$ with hole and electron capture cross sections $\sigma_{p}=6.0 \times 10^{-16} \mathrm{~cm}^{2}$ and $\sigma_{n}=1.60$ $\times 10^{-14} \mathrm{~cm}^{2}$, respectively. In addition to those studies, Rohatgi et al. ${ }^{4}$ applied DLTS coupled with dark and illuminated $\mathrm{I}-\mathrm{V}$ measurements and concluded the defect energy to be $E_{t}=E_{V}+0.30 \mathrm{eV}$. Hamaguchi et al. $^{5}$ applied DLTS and optical-DLTS to investigate the Mo-related defects in silicon and reported $E_{t}=E_{V}+0.31 \mathrm{eV}$. Similarly Pettersson et al. ${ }^{6}$ applied junction space charge techniques (JSCTs) and found $E_{t}=E_{V}+0.298 \mathrm{eV}$. More recently, Rein et al. ${ }^{7,8}$ applied a combination of temperature and injection dependent lifetime spectroscopy to determine $E_{t}=E_{V}+0.317 \pm 0.05 \mathrm{eV}$ and the ratio of the capture cross sections $\sigma_{n} / \sigma_{p}=13 \pm 3$. Furthermore, they concluded that both $\sigma_{p}$ and $\sigma_{n}$ have a dependence on temperature of $T^{-1.5}$, where it was assumed that the temperature dependence of $\sigma_{n} / \sigma_{p}$ was constant. In this work, we

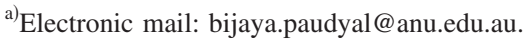

explicitly measure the temperature dependence of both $\sigma_{p}$ and $\sigma_{n}$, from which we also determine the temperature dependent trend of $\sigma_{n} / \sigma_{p}$. Furthermore, the measured temperature dependence of $\sigma_{n}$ is used to assess $E_{t}$ of Mo in silicon. Table I summarizes these published results on the interstitial Mo defect in Si.

This paper presents a novel and relatively simple technique to determine $\sigma_{p}(T)$ and $\sigma_{n}(T)$ over a wide range of temperature. It employs injection-dependent lifetime spectroscopy with a T-controlled photoconductance (PC) measurement instrument. Section II explains the carrier lifetime theory and its simplification in order to determine the temperature-dependent expression for $\sigma_{p}(T)$ and $\sigma_{n}(T)$, and the application of $\sigma_{n}(T)$ to determine $E_{t}$. This is followed by Sec. III that presents the preparation of Mo-contaminated samples for this work, the instrument and the details of the measurement procedure. In Sec. IV, we present $\sigma_{p}(T)$ and $\sigma_{n}(T)$, and analyze their trends to give insight into the effective capture mechanism and to assess $E_{t}$.

\section{THEORY}

The simplified version of Shockley Read Hall (SRH) lifetime ${ }^{9,10}$ for a single defect can be written as ${ }^{11}$

$$
\tau_{\mathrm{SRH}}=\frac{\tau_{n 0}\left(p_{1}+p_{0}+\Delta n\right)+\tau_{p 0}\left(n_{1}+n_{0}+\Delta n\right)}{\left(n_{0}+p_{0}+\Delta n\right)},
$$

where $\tau_{n 0}$ and $\tau_{p 0}$ are the fundamental capture time constants for electrons and holes, $n_{1}=N_{c} \exp \left[-\left(E_{c}-E_{t}\right) / k_{B} T\right]$ and $p_{1}$ $=N_{v} \exp \left[-\left(E_{t}-E_{v}\right) / k_{B} T\right], N_{c}$ and $N_{v}$ are the effective densities of states at the conduction and the valance band edge, $k_{B}$ is the Boltzmann's constant, $n_{0}$ and $p_{0}$ are the electron and hole densities at thermal equilibrium, and $\Delta n$ is the excess 
TABLE I. Summary of published results for interstitial molybdenum in crystalline silicon. The table lists the capture cross section of electrons $\sigma_{n}$ and holes $\sigma_{p}$, and the defect energy $E_{t}$ above the valence band edge $E_{v}$.

\begin{tabular}{lcccc}
\hline \hline $\begin{array}{l}\sigma_{p} \\
\left(\mathrm{~cm}^{2}\right)\end{array}$ & $\begin{array}{c}\sigma_{n} \\
\left(\mathrm{~cm}^{2}\right)\end{array}$ & $\sigma_{n} / \sigma_{p}$ at $27{ }^{\circ} \mathrm{C}$ & $\begin{array}{c}E_{t} \\
(\mathrm{eV})\end{array}$ & Ref. and technique \\
\hline $6.0 \times 10^{-16}$ at $27{ }^{\circ} \mathrm{C}$ & $1.6 \times 10^{-14}$ at $27^{\circ} \mathrm{C}$ & 26.67 & $E_{v}+0.28$ & DLTS, $^{\text {a }}$ average \\
$\mathrm{NA}$ & $\mathrm{NA}$ & $\mathrm{NA}$ & $E_{v}+0.31$ & DLTS $^{\mathrm{b}}$ \\
$\mathrm{NA}$ & $\mathrm{NA}$ & $\mathrm{NA}$ & $E_{v}+0.30$ & DLTS $^{\mathrm{c}}$ \\
$\mathrm{NA}$ & $7.8 \times 10^{-15}$ at $27^{\circ} \mathrm{C}$ & 13.0 & $E_{v}+0.317$ & TIDLS $^{\mathrm{d}}$ \\
$\mathrm{NA}$ & $\mathrm{NA}$ & $\mathrm{NA}$ & $E_{v}+0.298$ & JSCT $^{\mathrm{e}}$ \\
$6.05 \times 10^{-14} \times T^{-1.07}$ & $4.21 \times 10^{-8} \times T^{-2.95}$ & $11 \pm 2$ & $E_{v}+0.375 \pm 0.03$ & This work, TIDLS \\
\hline \hline
\end{tabular}

${ }^{\mathrm{a}}$ Reference 1.

${ }^{\mathrm{b}}$ Reference 5 .

${ }^{\mathrm{c}}$ Reference 4.

${ }^{\mathrm{d}}$ References 7 and 8 .

${ }^{\mathrm{e}}$ Reference 6.

carrier density. Equation (1) assumes that trapping effects are negligible (i.e., $\Delta n=\Delta p)^{11}$

For a $p$-type wafer, $n_{0}$ can be neglected in comparison with $p_{0}$ and when $\Delta n$ is sufficiently less than $p_{0}, \tau_{\mathrm{SRH}}$ can be rewritten as

$$
\tau_{\mathrm{SRH}}=\frac{\tau_{n 0}\left(p_{1}+p_{0}\right)+\tau_{p 0}\left(n_{1}+n_{0}\right)}{p_{0}}+\frac{\tau_{p 0}}{p_{0}} \Delta n .
$$

At a sufficiently low temperature, emission from the defect energy level is negligible and $n_{1}(T)+n_{0}(T) \ll \Delta n$ and $p_{1}(T)$ $\ll p_{0}(T)$. In such a case, Eq. (2) can be simplified further to become

$$
\tau_{\mathrm{SRH}}=\tau_{n 0}+\frac{\tau_{p 0}}{p_{0}} \times \Delta n
$$

Figure 1 presents a plot that shows the temperature below which Eq. (3) is valid. It plots $T$ when $n_{1}+n_{0}=0.01 \times \Delta n$ $=0.0001 \times p_{0}$ and when $p_{1}=0.01 \times p_{0}$ as a function of $E_{t}$ and $N_{A}$. It assumes $n_{1}+n_{0}$ negligible in comparison to $\Delta n$ when earlier's value is less than $1 \%$ of the latter and a similar assumption applies to $p_{1}$ and $p_{0}$. At temperatures below the lines in Fig. 1 (for a given $N_{A}$ ), it is safe to employ Eq. (3). The figure shows that the temperature below which both assumptions are valid decreases as (i) $N_{A}$ decreases and (ii) as the defect energy level moves further from midgap. The latter occurs because thermal emission from a defect level to a

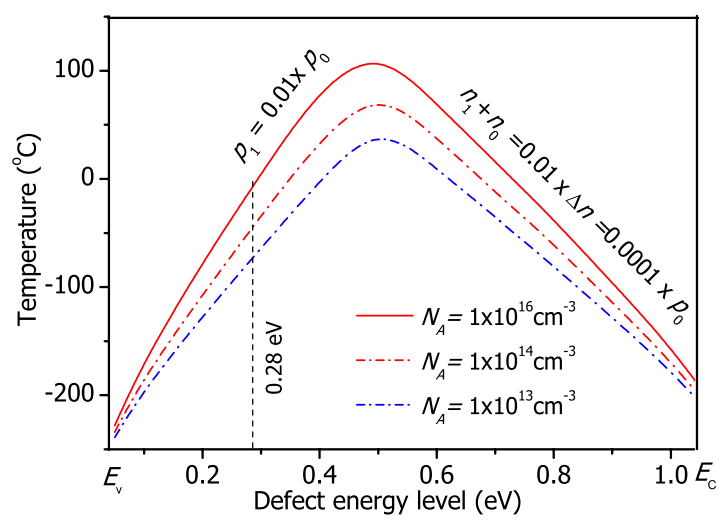

FIG. 1. (Color online) Temperature below which $n_{1}+n_{0}=0.01 \times \Delta n$ $=0.0001 \times p_{0}$ and $p_{1}=0.01 \times p_{0}$ as a function of $E_{t}$ for $p$-type silicon of three doping densities $\left(N_{A}\right)$. band edge is high when the defect energy is near that band edge.

At temperatures where Eq. (3) is valid, the slope of a plot of $\tau_{\mathrm{SRH}}$ against $\Delta n$ gives $\tau_{p 0} / p_{0}$ and the intercept gives $\tau_{n 0}$. Hence $\sigma_{n}$ and $\sigma_{p}$ can be calculated using the measured slope and the intercept of a linear plot of $\tau_{\mathrm{SRH}}$ against $\Delta n$ for different temperatures, as

$$
\begin{gathered}
\sigma_{p}=\frac{1}{\tau_{p 0} v_{t h p} N_{t}}=\frac{1}{\text { Slope } \times p_{0} v_{t h p} N_{t}}, \\
\sigma_{n}=\frac{1}{\tau_{n 0} v_{t h n} N_{t}}=\frac{1}{\text { Intercept } \times v_{t h n} N_{t}},
\end{gathered}
$$

where $v_{t h p}$ and $v_{t h n}$ are the thermal velocities of holes and electrons and $N_{t}$ is the defect concentration. In some cases the effective capture mechanism can be inferred from the temperature dependence of $\sigma_{n}(T)$ and $\sigma_{p}(T){ }^{12-14}$

At higher temperatures, when carrier emission from the defect energy level is significant and $n_{1}(T)+n_{0}(T)$ is not negligible compared with $\Delta n$ or $p_{1}(T)$ is not negligible in comparison to $p_{0}(T)$, the more generalized expression of Eq. (2) must be employed. In such a case, the slope of a plot of $\tau_{\mathrm{SRH}}$ against $\Delta n$ is still $\tau_{p 0}(T)$ and Eq. (4) remains a valid way to determine $\sigma_{p}(T)$. However, the intercept of the plot becomes a function of $p_{1}(T)$ and/or $n_{1}(T)$, which are dependent upon $E_{t}$. Therefore, a value of $E_{t}$ is required to calculate $\sigma_{n}(T)$ at higher temperatures. By extrapolating the observed trend of $\sigma_{n}(T)$ measured at lower temperatures, and assuming the dominant capture mechanism remains unchanged as the temperature increases, it is therefore possible to determine the energy level itself.

\section{EXPERIMENT}

A silicon ingot was grown with the pedestal growth technique and boron-doped with a resistivity of $1.8 \Omega \mathrm{cm}$. The ingot was intentionally contaminated by adding 2800 ppma of molybdenum (Mo) in the silicon melt. ${ }^{15}$ A Mo concentration of $2 \times 10^{13} \mathrm{~cm}^{-3}$ was determined by neutron activation analysis (NAA) on the section of the ingot studied here, as described in detail elsewhere. ${ }^{15}$ If all of the Mo atoms are interstitial and active, this gives an upper limit to $N_{t}$ of 2 $\times 10^{13} \mathrm{~cm}^{-3}$. The experiment was performed on a silicon 


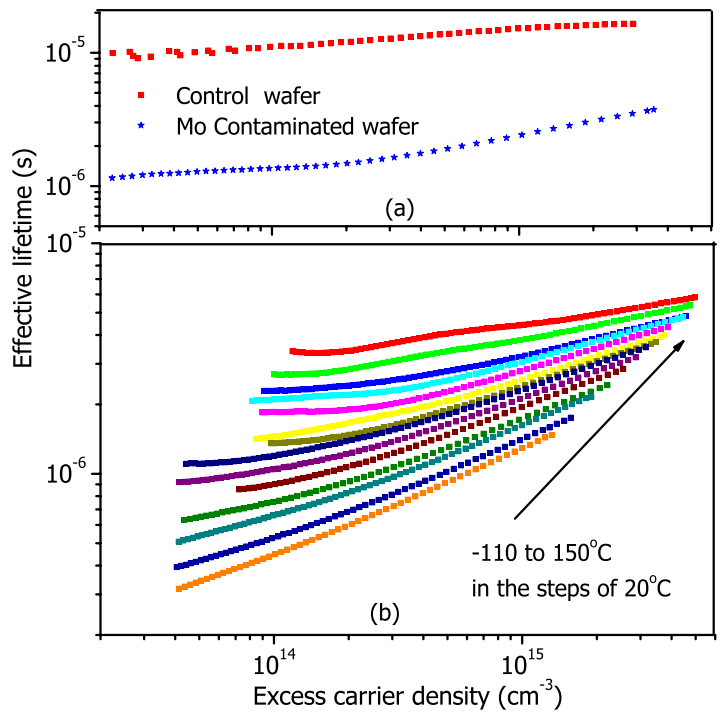

FIG. 2. (Color online) (a) Measured lifetime of the Mo-contaminated and control wafers at room temperature. (b) Measured lifetime of Mocontaminated wafer for temperatures of -110 to $150{ }^{\circ} \mathrm{C}$.

sample sliced from the ingot. The wafer was subject to a phosphorus diffusion step, to remove unintended fastdiffusing impurities such as Fe, which may otherwise affect the lifetime. The diffused layers were then removed by chemical polishing, followed by cleaning and a plasma enhanced-chemical vapor deposited, $\mathrm{SiN}_{\mathrm{x}}$ layer optimized for surface passivation as described by Coletti et al. ${ }^{16}$ The average width of the sample was $285 \mu \mathrm{m}$.

Lifetime measurements were performed on a temperature controlled inductive coil PC based instrument, described in detail elsewhere. ${ }^{17}$ Figure 2(a) depicts the injection dependent lifetime data for the Mo-contaminated wafer and a control wafer at room temperature, and Fig. 2(b) presents injection dependent lifetime data for the Mo-contaminated wafer for -110 to $150{ }^{\circ} \mathrm{C}$. The effective lifetime $\left(\tau_{\text {eff }}\right)$ in the Mocontaminated wafer was found to be an order of magnitude smaller than the control wafer, which indicates that the Mo impurities have a dominant effect on overall recombination. We therefore assume the measured quantity $\tau_{\text {eff }}$ is equal to the $\tau_{\mathrm{SRH}}$ that is associated with interstitial Mo in these samples.

The quasisteady-state PC technique ${ }^{18}$ was employed to

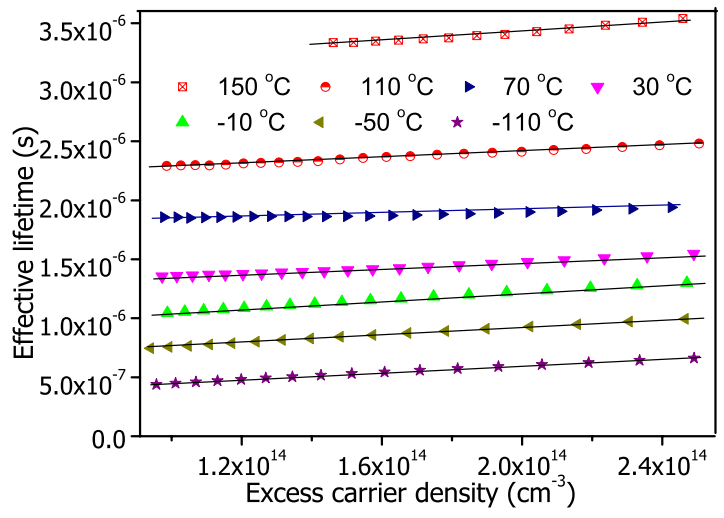

FIG. 3. (Color online) Lifetime plot and linear fit of Mo-doped $(1.8 \Omega \mathrm{cm}$ ) wafer for the selected injection range $\left(1 \times 10^{14}-2 . \times 10^{14} \mathrm{~cm}^{-3}\right)$.

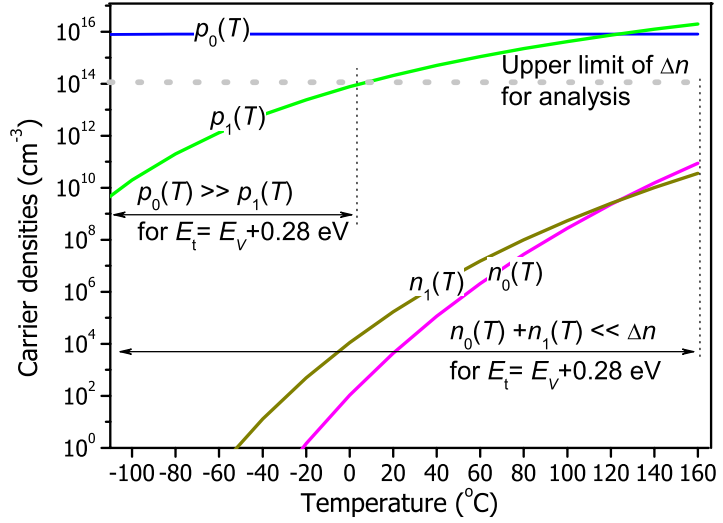

FIG. 4. (Color online) Carrier densities $\left(n_{1}, p_{1}, n_{0}\right.$, and $\left.p_{0}\right)$ for a defect energy level $\left(E_{V}+0.28 \mathrm{eV}\right)$.

determine the carrier lifetime at different temperatures. The carrier mobility model developed by Reggiani et al. ${ }^{19}$ was applied for the subsequent analysis of the measured lifetime data. This mobility model accounts for the effect of temperature and dopant concentration but does not account for the effect of $\Delta n$. The Reggiani model for carrier mobility was therefore modified by replacing the donor density $N_{D}$ with $N_{D}+\Delta n$ and the acceptor density $N_{A}$ with $N_{A}+\Delta n$. This approximation was found to give a good agreement with the mobility model of Klaassen et al. ${ }^{20,21}$ at room temperature. Reggiani's carrier mobility model was preferred in this case because of it validity over a larger temperature range ( -73 to $327^{\circ} \mathrm{C}$ ). The temperature across the wafers was found to vary by $\pm 2 \%$ during measurement and the uncertainty in the measured lifetime was $\pm 6 \%,{ }^{17}$ which depends mostly upon the calibration of the illumination intensity and the inductive coil. The effective lifetime $\tau_{\text {eff }}$ was measured as a function of $\Delta n$ over a temperature range of -110 to $150{ }^{\circ} \mathrm{C}$ at intervals of $10{ }^{\circ} \mathrm{C}$. Figure 3 depicts the measured $\tau_{\text {eff }}$ as a function of $\Delta n$ from -110 to $150{ }^{\circ} \mathrm{C}$ in steps of $40{ }^{\circ} \mathrm{C}$.

When $\Delta n<2.5 \times 10^{14} \mathrm{~cm}^{-3}$, the above mentioned conditions of Eqs. (2) and (3) are satisfied. The carrier densities $\left(n_{1}, p_{1}, n_{0}\right.$, and $\left.p_{0}\right)$ and $\Delta n$ for the analysis are depicted in Fig. 4 for the defect energy level of $E_{V}+0.28 \mathrm{eV}$ in silicon. This graph shows $n_{1}+n_{0} \ll 1 \times 10^{14} \mathrm{~cm}^{-3}$ for temperatures less than $160^{\circ} \mathrm{C}$ and $p_{1} \ll p_{0}$ for temperatures less than $0{ }^{\circ} \mathrm{C}$. Table II depicts the temperature ranges for which the assumption $\left(n_{1}+n_{0}\right) \ll \Delta n$ and $p_{1} \ll p_{0}$ is valid for different reported values of $E_{t}$, where we require $\left(n_{1}+n_{0}\right)$ and $p_{1}$ to be no more than $1 \%$ of $\Delta n$ and $p_{0}$, respectively. In this work, we choose the most conservative upper limit of temperature for the subsequent analysis, as represented by the vertical line in Fig. 1. $n_{0}(T)$ was determined by using the doping density

TABLE II. Upper limit of $T_{\text {low }}\left({ }^{\circ} \mathrm{C}\right)$ for which the assumptions $\left(n_{1}+n_{0}\right)$ $<0.01 \times \Delta n$ and $p_{1}<0.01 \times p_{0}$ is valid for analysis.

\begin{tabular}{lccc}
\hline \hline $\begin{array}{l}E_{t} \\
(\mathrm{eV})\end{array}$ & $\begin{array}{c}\left(n_{1}+n_{0}\right) \ll \Delta n \\
\left({ }^{\circ} \mathrm{C}\right)\end{array}$ & $\begin{array}{c}p_{1} \ll p_{0} \\
\left({ }^{\circ} \mathrm{C}\right)\end{array}$ & Ref. \\
\hline$E_{V}+0.28$ & $T \leq 160$ & $T \leq 0$ & 1 \\
$E_{V}+0.30$ & $T \leq 158$ & $T \leq 10$ & 4 \\
$E_{V}+0.317$ & $T \leq 155$ & $T \leq 18$ & 7 and 8 \\
\hline \hline
\end{tabular}




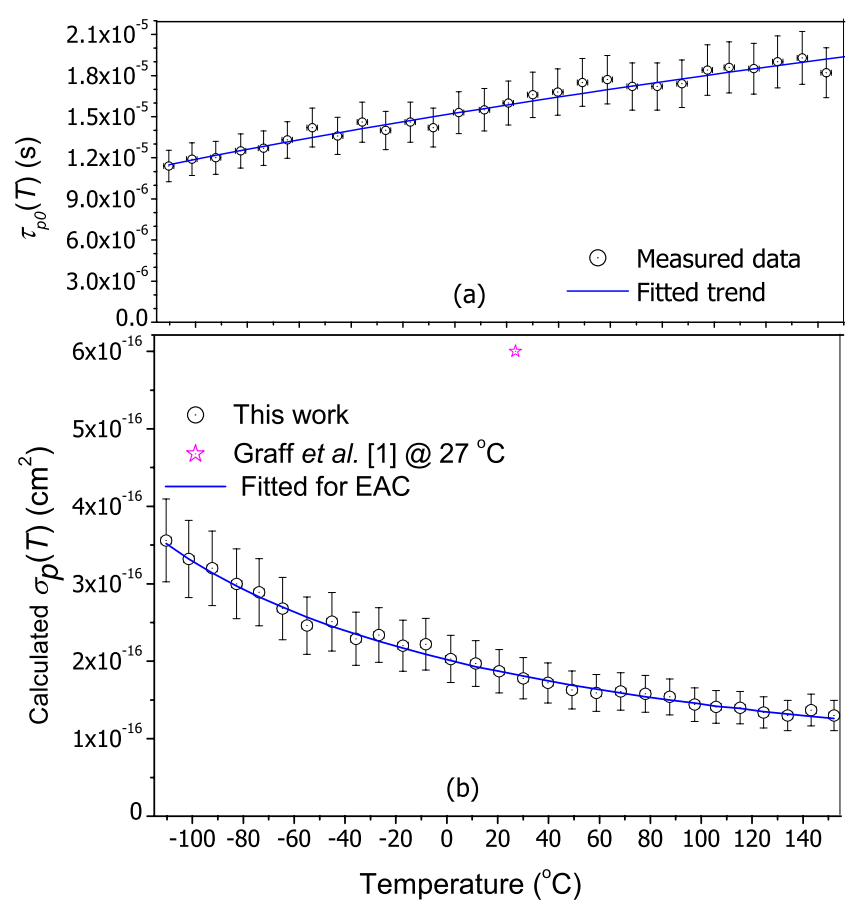

FIG. 5. (Color online) Measured $\tau_{p 0}$ (a) and calculated $\sigma_{p}(T)$ for Mo impurities in silicon (b) with an EAC mechanism fit, depicting other previously published values at RT.

$\left(N_{A}\right)$ and the temperature dependent intrinsic carrier concentration, $n_{i}(T) .{ }^{22}$ Freeze-out of the boron atoms in the wafer was taken into account at the low temperature $\left(<77^{\circ} \mathrm{C}\right)$ by adopting the T-dependent model of $p_{0}(T)$ from Ashcroft and Mermin. ${ }^{23}$ T-dependent models for $v_{t h n}$ and $v_{t h p}$ reported by Green et $a l^{22}$ were employed to calculate $\sigma_{n}$ and $\sigma_{p}$ using Eqs. (4) and (5).

\section{RESULTS AND DISCUSSION}

Figure 5(a) plots the measured $\tau_{p 0}(T)$ over the temperature range of -110 to $150{ }^{\circ} \mathrm{C}$ and Fig. 5(b) plots $\sigma_{p}(T)$ calculated with Eq. (4) assuming that $N_{t}$ equals the Mo concentration. The figure shows that $\sigma_{p}$ decreases with temperature over the entire temperature range. This eliminates the possibility that hole capture occurs via multiphonon emission in which $\sigma_{p}$ increases with temperature. ${ }^{12}$ We also rule out cascade capture as a possible capture mechanism because it is only relevant to shallow Coulomb attractive defect centers. ${ }^{14}$ This leaves excitonic Auger capture (EAC) (Ref. 13) as the most likely mechanism for the capture of holes by the Mo defect.

The temperature dependence of $\sigma$ under EAC is governed by the equation, $\sigma=\sigma_{0} T^{\alpha}$, where $\sigma_{0}$ and $\alpha$ are independent of temperature. The line in Fig. 5 is a fit of this equation to the experimental data, where $\sigma_{0}=6.05 \pm 0.8$ $\times 10^{-14} \mathrm{~cm}^{-2}$ and $\alpha=-1.07 \pm 0.05$. The uncertainty in these values represents a 95\% confidence interval from an errorweighted least-square fit to the experimental data.

The above calculation assumes $100 \%$ of the Mo atoms, determined by NAA measurements, are electrically active, however this may not be valid if, for example, precipitation occurs $^{1}$ or in case of uncertainties in NAA measurement. In

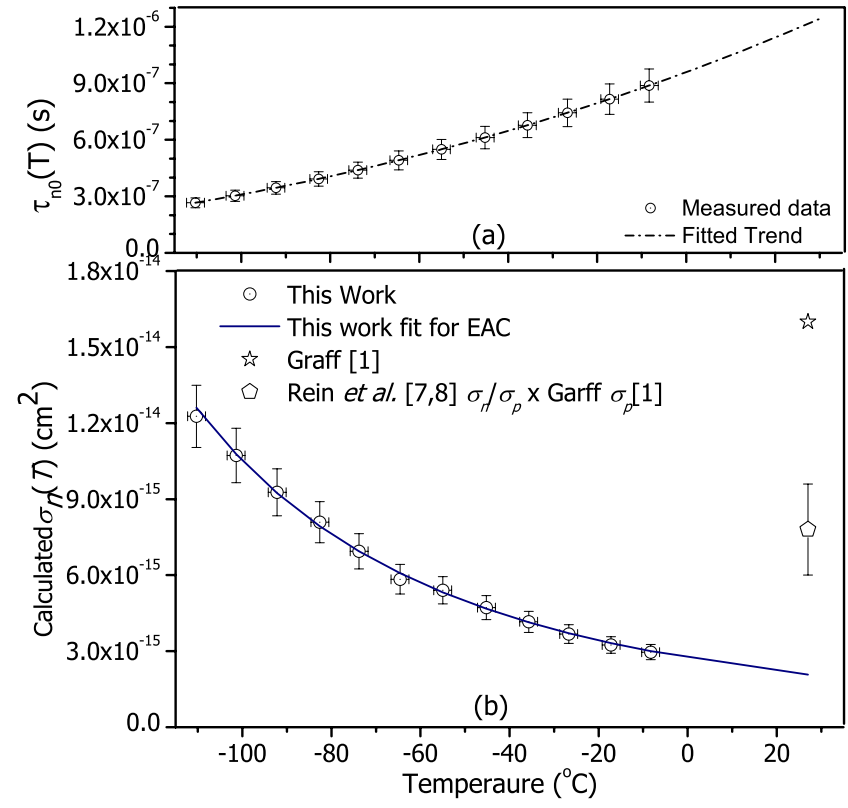

FIG. 6. (Color online) Measured $\tau_{n 0}$ (a) and calculated $\sigma_{n}(T)$ for Mo impurities in silicon (b) with an EAC mechanism fit, depicting other previously published values at RT.

the case where only a fraction $f_{\text {act }}$ of Mo atoms are electrically active, the temperature dependence of the hole capture cross section is therefore

$$
\sigma_{p}(T)=\frac{1}{f_{\text {act }}}\left(6.05 \pm 0.8 \times 10^{-14}\right) T^{-(1.07 \pm 0.05)} .
$$

Thus, $\sigma_{0}$ is inversely proportional to $f_{\text {act }}$, while $\alpha$ is independent of $f_{\text {act }}$. If the fraction of electrically active impurities were $f_{\text {act }}=0.3, \sigma_{p}$ becomes consistent with the DLTS value reported by Graff at room temperature. ${ }^{1}$ We note that a fractional activation of Mo atoms does not alter the conclusion that EAC is the most likely capture mechanism of holes.

The electron capture cross section $\sigma_{n}(T)$ was first determined for the temperature range of -110 to $0{ }^{\circ} \mathrm{C}$ by implementing Eq. (5), which does not require $E_{t} . \sigma_{n}$ is also found to decrease monotonically and like $\sigma_{p}$, it can be best described by an EAC mechanism. The EAC fit of the measured $\sigma_{n}$ gives

$$
\sigma_{n}(T)=\frac{1}{f_{\text {act }}}\left(4.21 \pm 0.4 \times 10^{-8}\right) T^{-(2.95 \pm 0.02)} .
$$

Figure 6(a) shows the measured $\tau_{n 0}(T)$ over the temperature range of -110 to $0{ }^{\circ} \mathrm{C}$ and Fig. $6(\mathrm{~b})$ shows the calculated $\sigma_{n}(T)$ when $f_{\text {act }}=1$. When $f_{\text {act }}=0.3, \sigma_{n}$ extrapolated to room temperature is consistent with Rein's value of $\sigma_{n} / \sigma_{p}$ multiplied by Graff's value of $\sigma_{p}$, and when $f_{\text {act }}=0.15, \sigma_{n}$ is consistent with Graff's value of $\sigma_{n}$.

Temperature-dependent values of $\sigma_{n}$ for the higher temperature range $\left(0-150{ }^{\circ} \mathrm{C}\right)$ were then calculated using various reported values of $E_{t}$ for Mo ${ }^{1,4,7,8}$ as required in Eq. (2). Figure 7(a) depicts the measured intercepts and Fig. 7(b) shows the corresponding $\sigma_{n}(T)$ calculated for different $E_{t}$. This reveals that an energy level of $E_{t}=E_{V}$ $+0.375 \pm 0.03 \mathrm{eV}$ is required to fit the measured intercept for the extended trend of $\sigma_{n}(T)$ when we assume the EAC 

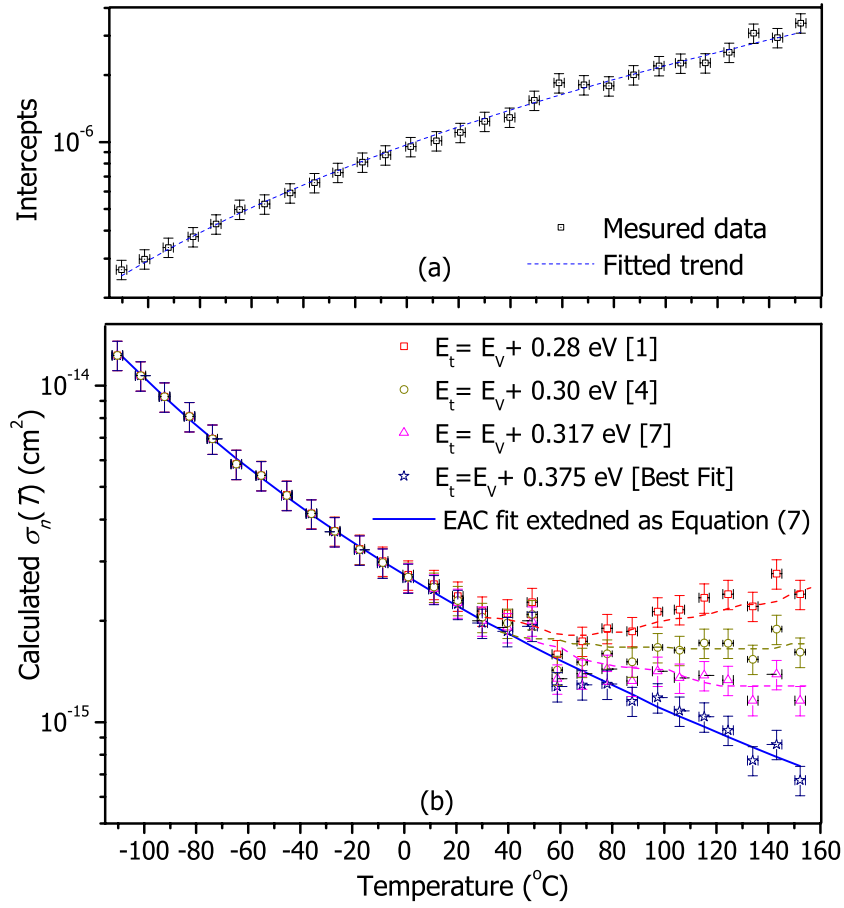

FIG. 7. (Color online) Measured intercepts of plot ( $\tau_{\text {eff }}$ vs $\Delta n$ ) (a) and calculated $\sigma_{n}(T)$ for Mo for previously reported values of $E_{t}$. Depicts the best fit with $E_{t}=E_{v}+0.375 \mathrm{eV}$ for EAC mechanism.

mechanism described by Eq. (7) remains dominant. It is possible, however, that the EAC mechanism does not remain dominant and another recombination mechanism that causes $\sigma_{n}(T)$ to increase with temperatures becomes significant at temperatures above $0{ }^{\circ} \mathrm{C}$. We therefore do not discount the possibility that $E_{t}$ is smaller than $E_{v}+0.375 \mathrm{eV}$ and closer to values determined by other authors $1,4,7,8$

Figure 8 plots the ratio of the capture cross sections $\left(\sigma_{n} / \sigma_{p}\right)$ over the temperature range, -110 to $150{ }^{\circ} \mathrm{C}$. The symbols in Fig. 8 plot the data assuming $E_{t}=E_{v}+0.375 \mathrm{eV}$, and the error bars combine the uncertainty in the measurement with the uncertainty in $E_{t}$. The figure shows that $\sigma_{n} / \sigma_{p}$ decreases with temperature, even though both carriers are best described by the same capture mechanism (EAC). The data agrees well with the value reported by Rein $e t a l{ }^{7,8}$ at

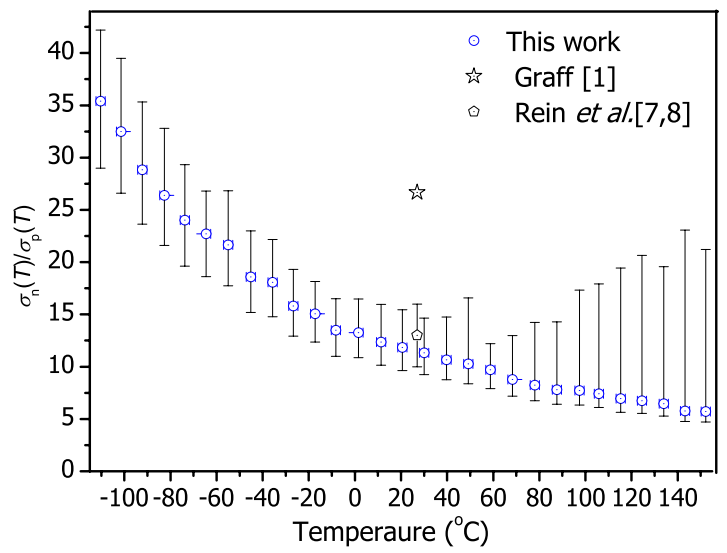

FIG. 8. (Color online) Capture cross section ratio of Mo defect in silicon at different temperatures. room temperature. The uncertainty due to any partial activation of Mo atoms in silicon [represented by $f_{\text {act }}$ in Eqs. (6) and (7)] cancels in the calculation of $\sigma_{n} / \sigma_{p}$. However the uncertainty in $\sigma_{n} / \sigma_{p}$ increases at higher temperature $\left(>0{ }^{\circ} \mathrm{C}\right)$ due to the uncertainty in $\sigma_{n}$ associated with the uncertainty in $E_{t}$.

\section{CONCLUSIONS}

Temperature-dependent expressions for $\sigma_{n}$ and $\sigma_{p}$ of interstitial molybdenum in silicon were independently determined over the temperature range, -110 to $150{ }^{\circ} \mathrm{C}$, using a temperature controlled PC instrument. The T-dependent trends of $\sigma_{p}$ and $\sigma_{n}$ are best matched by an EAC mechanism, giving minimum values of the coefficients of $\sigma_{0}=6.1 \pm 0.8$ $\times 10^{-14}$ and $4.2 \pm 0.4 \times 10^{-8} \mathrm{~cm}^{2}$ and giving T-exponents of $\alpha=1.07 \pm 0.05$ and $2.95 \pm 0.2$. Assuming the EAC mechanism also dominates electron capture at higher temperatures, the energy level of interstitial Mo in silicon is determined to be $0.375 \pm 0.03 \mathrm{eV}$ above the valance band of silicon.

\section{ACKNOWLEDGMENTS}

D.M. likes to thank the Australian Research Council for fellowship and G.C. likes to thank "CrystalClear Integrated Project" (Contract No. SES6-CT_2003-502583) funded by the European Commission.

${ }^{1}$ K. Graff, Metal Impurities in Silicon-Device Fabrication (Springer-Verlag, Berlin, 1995).

${ }^{2}$ A. A. Istratov, T. Buonassisi, R. J. McDonald, A. R. Smith, R. Schindler, J. A. Rand, J. P. Kalejs, and E. R. Weber, J. Appl. Phys. 94, 6552 (2003).

${ }^{3}$ J. R. Davis, Jr., A. Rohatgi, R. H. Hopkins, P. D. Blais, P. Rai-Choudhury, J. R. McCormick, and H. C. Mollenkopf, IEEE Trans. Electron Devices 27, 677 (1980).

${ }^{4}$ A. Rohatgi, R. H. Hopkins, J. R. Davis, R. B. Campbell, and H. C. Mollenkopf, Solid-State Electron. 23, 1185 (1980).

${ }^{5}$ T. Hamaguchi and Y. Hayamizu, Jpn. J. Appl. Phys., Part 2 30, L1837 (1991).

${ }^{6}$ H. Pettersson, H. G. Grimmeiss, L. Tilly, K. Schmalz, K. Tittelbach, and H. Kerkow, Semicond. Sci. Technol. 6, 237 (1991).

${ }^{7}$ S. Rein, Lifetime Spectroscopy: A Method of Defect Characterization in Silicon for Photovoltaic Applications (Springer, New York, 2005).

${ }^{8}$ S. Rein, S. Diez, and S. W. Glunz, Proceedings of the 19th EC PVSEC, Paris, France, 2004 (unpublished), pp. 219-222.

${ }^{9}$ W. Shockley and W. T. Read, Phys. Rev. 87, 835 (1952).

${ }^{10}$ R. N. Hall, Phys. Rev. 87, 387 (1952).

${ }^{11}$ A. G. Aberle, The University of New South Wales (1999).

${ }^{12}$ C. H. Henry and D. V. Lang, Phys. Rev. B 15, 989 (1977).

${ }^{13}$ A. Hangleiter, Phys. Rev. B 37, 2594 (1988).

${ }^{14}$ M. Lax, Phys. Rev. 119, 1502 (1960).

${ }^{15}$ G. Coletti, L. J. Geerligs, P. Manshanden, C. Swanson, S. Riepe, W. Warta, J. Arumughan, and R. Kopecek, Solid State Phenomena 131, 15 (2008).

${ }^{16}$ G. Coletti, R. Kvande, V. D. Mihailetchi, L. J. Geerligs, L. Arnberg, and E. J. Øvrelid, J. Appl. Phys. 104, 104913 (2008).

${ }^{17}$ B. B. Paudyal, K. R. McIntosh, D. H. Macdonald, B. S. Richards, and R. A. Sinton, Prog. Photovoltaics 16, 609 (2008).

${ }^{18}$ R. A. Sinton and A. Cuevas, Appl. Phys. Lett. 69, 2510 (1996).

${ }^{19}$ S. Reggiani, M. Valdinoci, L. Colalongo, M. Rudan, G. Baccarani, A. D. Stricker, F. Illien, N. Felber, W. Fichtner, and L. Zullino, IEEE Trans. Electron Devices 49, 490 (2002).

${ }^{20}$ D. B. M. Klaassen, Solid-State Electron. 35, 953 (1992).

${ }^{21}$ D. B. M. Klaassen, Solid-State Electron. 35, 961 (1992).

${ }^{22}$ M. A. Green, J. Appl. Phys. 67, 2944 (1990).

${ }^{23}$ N. W. Ashcroft and N. D. Mermin, Saunders College, Philadelphia (1976). 